\title{
Dictynna
}

Dictynna

Revue de poétique latine

2| 2005

Varia

\section{Il passato come nemico: Annibale e la velleitaria lotta contro una storia esemplare}

\section{Marco Fucecchi}

\section{(2) OpenEdition}

1 Journals

\section{Edizione digitale}

URL: http://journals.openedition.org/dictynna/127

DOI: $10.4000 /$ dictynna. 127

ISSN: $1765-3142$

\section{Edizione cartacea}

ISBN: 978-2-84467-104-2

\section{Notizia bibliografica digitale}

Marco Fucecchi, « II passato come nemico: Annibale e la velleitaria lotta contro una storia esemplare », Dictynna [En ligne], 2 | 2005, mis en ligne le 30 novembre 2010, consulté le 10 septembre 2020. URL : http://journals.openedition.org/dictynna/127 ; DOI : https://doi.org/10.4000/dictynna.127

Questo documento è stato generato automaticamente il 10 settembre 2020.

\section{(c) (i) (9)}

Les contenus des la revue Dictynna sont mis à disposition selon les termes de la Licence Creative Commons Attribution - Pas d'Utilisation Commerciale - Pas de Modification 4.0 International. 


\title{
Il passato come nemico: Annibale e la velleitaria lotta contro una storia esemplare
}

\author{
Marco Fucecchi
}

1 La rapida avanzata di Annibale nel cuore dell'Italia acquista - com'è noto - un rilievo particolare nel racconto di Silio Italico, che dedica più della metà dei 17 libri dei Punica alla trattazione degli eventi compresi tra il 218 e il 216, l'anno della battaglia di Canne ${ }^{1}$. I presupposti estetico-ideologici di una scelta che privilegia, di fatto, la rappresentazione delle sconfitte più brucianti subite da Roma nella penisola ${ }^{2}$ sono stati giustamente individuati nella tendenza (che Silio condivide con Livio, ma che era già stata elaborata dalla cultura romana di età tardo-repubblicana) ad esaltare il significato della seconda guerra punica come momento di svolta epocale: la prova più difficile affrontata da una comunità ambiziosa, che facendo appello a tutte le sue risorse umane, morali ed economiche era riuscita a ribaltare una situazione disperata, ottenendo un'affermazione che l'avrebbe portata a ricoprire un ruolo egemone nel Mediterraneo ${ }^{3}$.

2 La serie di cruente battaglie, che si avvicendano con ritmo incalzante (e, invero, un po' monotono) davanti agli occhi del lettore, mette in risalto l'efficacia della macchina militare cartaginese e le qualità indiscutibili del suo condottiero. La figura isolata di Annibale fa retrocedere in secondo piano quelle dei consoli romani che si alternano, con esito sfortunato, nel vano tentativo di opporgli resistenza. A questi ultimi, d'altra parte, non si può complessivamente rimproverare mancanza di coraggio o di valore (l'unica eccezione è il Terenzio Varrone di Canne). Publio Cornelio Scipione padre, per es., si rende protagonista di aristie notevoli, talora ingigantite nelle proporzioni dall'inventiva del poeta epico ${ }^{4}$. Ma anche un personaggio controverso come Gaio Flaminio, generale dell'esercito romano al Trasimeno, riesce a riscattare in buona parte il suo passato di demagogo con una morte eroica sul campo ${ }^{5}$. Infine, il comportamento di Emilio Paolo a Canne costituisce, addirittura, una sublimazione del gesto dell'autosacrificio: vittima incolpevole della scriteriata frenesia bellicista di un collega inetto (il Varrone di cui sopra), Paolo è costretto dal precipitare degli eventi a 
rinunciare alla prudentia strategica (una dote che lo distingue da predecessori come Flaminio o lo stesso Scipione padre, e la cui validità era stata poco prima dimostrata da Fabio Massimo), immolando se stesso al fine di ritardare quanto meno la vittoria di Annibale (10, 292 ss.).

Silio Italico, insomma, non risparmia alcuna delle risorse in suo possesso al fine di addensare il pathos della tragedia, da cui deve programmaticamente risaltare 'e contrario' il significato emblematico e didattico delle sconfitte romane. Al tempo stesso, tuttavia, il testo si incarica di relativizzare gli effetti di questo quadro desolante evidenziando a più riprese il saldo e provvidenziale controllo esercitato da Giove sull'intera vicenda: dopo aver rassicurato Venere all'immediata vigilia della campagna militare di Annibale in Italia (3, 571 ss. pelle metus ... / ..., Cytherea; tenet longumque tenebit / Tarpeias arces sanguis tuus), il dio prende nuovamente la parola dopo la sconfitta del Trasimeno per sancire l'inviolabilità di Roma $(6,600$ ss.), e poi, verso la fine della battaglia di Canne, per annunciare l'avvento ormai imminente del giovane Scipione ( 9 , 543 ss.); fino al momento in cui - insieme agli altri abitatori del nuovo olimpo (i colli di Roma dove hanno sede i templi delle divinità) - egli ingaggerà personalmente una battaglia vittoriosa contro il 'titano' cartaginese (12, $605 \mathrm{ss}$.).

Ma a giocare contro Annibale non c'è solo il futuro immediato di questo conflitto, destinato ad evolversi in un senso a lui sfavorevole. Il luminoso destino di Roma, di cui la vittoria nella seconda guerra punica costituisce una tappa fondamentale, affonda già le sue radici nel passato della città. Ed è proprio il 'ritorno del passato', la valorizzazione attiva della sua permanente efficacia, che nel testo di Silio caratterizza la lunga e angosciosa attesa di una svolta, contribuendo a smorzare l'oscurità dei momenti più difficili e a dare idealmente ai protagonisti romani e al lettore il coraggio di resistere e sperare.

5 La scelta di un tema antico come la guerra contro Cartagine permette, da un lato, al poeta flavio di avvalersi della tecnica virgiliana di anticipare il futuro dal passato con repentini sguardi in avanti sul domani più prossimo ${ }^{6}$ e su quello più lontano ${ }^{7}$. D'altra parte, la stessa scelta gli garantisce anche la possibilità di guardare indietro, di trarre cioè dal presente del racconto lo spunto per rintracciare nel passato analoghe situazioni in cui Roma, prima di vincere, aveva saputo soprattutto 'soffrire'. È proprio l'adozione di questa prospettiva retrograda, tipica dell'epos storico nazionale (che guarda al passato dal presente e che, dopo Lucano, torna a farlo in funzione eminentemente celebrativa), a favorire un recupero selettivo, mirato ed 'esemplare', della precedente storia di Roma.

6 Tale procedimento si concretizza in segmenti digressivi di natura ed estensione variabili (ekphraseis, racconti inseriti ecc.) che trovano portavoci e destinatari interni in alcuni protagonisti della narrazione principale (romani e no): di questi possono illustrare i trascorsi familiari o personali, e quindi motivarne le istanze; esprimerne o, in alcuni casi, addirittura contrastarne le aspirazioni individuali e le attese. Il quadro che ne scaturisce, perciò, non è sempre lineare: pur senza perdere la sua coerenza di fondo, garantita in ultima analisi dalla 'voce del testo' (il commento 'patriottico' del narratore primo), la rievocazione storica si presenta talvolta frammentata in una dialettica di posizioni e motivazioni, che riflettono la contrapposizione di forze in campo, e rivelano implicitamente la spiccata disponibilità dei Punica a tematizzare il problema dell'interpretazione del passato e della sua valenza propagandistica. 
7 I numerosi richiami alla prima guerra punica, ovvero l'evento che costituisce il presupposto immediato della materia della narrazione, illustrano in modo significativo questa varietà di atteggiamenti. Il lungo inserto metadiegetico che occupa gran parte del libro VI con la vicenda di Atilio Regolo raccontata dal vecchio commilitone Maro a un figlio dell'eroico e sfortunato generale, Serrano (un superstite della battaglia del Trasimeno), esemplifica degnamente la funzione edificante e consolatrice che la memoria del passato svolge sia presso il destinatario interno che, a un secondo livello, presso il pubblico dei lettori. Ma ancor più indicativo è il modo in cui le immagini e $\mathrm{i}$ destini dello stesso Regolo, da una parte, e, dall'altra, di Amilcare Barca (anche lui un personaggio-simbolo della prima guerra punica) vengono riproposti via via secondo

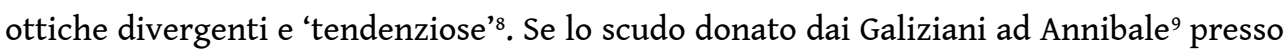
Sagunto (2, 406-452) ritrae Amilcare che imperversa ancora minaccioso nei territori di Sicilia (429 ss.), mentre Regolo vi figura tristemente appeso alla croce del supplizio (435 s.), le pitture di un tempio romano a Literno - che lo stesso condottiero cartaginese contempla prima di distruggerle in un accesso di furore $(6,653-697)$ - presentano uno spettacolo di tenore opposto ${ }^{10}$ : Regolo, convinto ispiratore della guerra, è protagonista di numerose vittorie in Africa ${ }^{11}$; Amilcare invece, mestamente raffigurato in catene, spicca quale primario oggetto di attrazione nella processione trionfale romana ${ }^{12}$.

Una simile dialettica di fonti e, soprattutto, di 'interpretazioni' parziali non significa, comunque, la rinuncia del narratore a prendere posizione: la reazione iconoclasta di Annibale e il suo vaneggiare un programma iconografico alternativo, basato sulle imprese appena compiute e quelle auspicate (Roma incendiata e Giove detronizzato) ${ }^{13}$, sono stati già negati e delegittimati, prima ancora di essere esplicitati, dalle parole di Giove stesso (6, 600 ss. ... "haud umquam tibi Iuppiter" inquit / "o iuvenis, dederit portas transcendere Romae / atque inferre pedem. ... (...) ... Tarpeium accedere collem / murisque adspirare veto").

Qualcosa di analogo per certi versi si verifica anche a proposito di ricordi provenienti dal passato più lontano: la discesa dei Galli Senoni guidati da Brenno, la loro vittoria presso il fiume Allia (intorno al 390 a. C.) e, a seguire, l'invasione di Roma e l'estenuante assedio del Campidoglio, il luogo dell'ultima resistenza. L'onta della sconfitta militare rimane nell'immaginario romano il punto forse più dolente ${ }^{14}$ : secondo la versione 'nazionalistica' offerta da Tito Livio $(5,48 \mathrm{~s}$.), infatti, la successiva presa della città sarebbe stata vendicata dal ritorno tempestivo di Camillo da Ardea, prima ancora che fosse pagato il riscatto chiesto dai Galli per abbandonare l'assedio del Campidoglio ${ }^{15}$. Occorre notare che nei Punica la voce narrante ricorda più volte proprio la disfatta dell'Allia, come modello 'superato' dalla gravità delle prime sconfitte subite dai consoli (per es. 1, 547 nec, Thrasymenne, tuis nuc Allia cederet undis; 6, 555 s. Allia et infandi Senones captaeque recursat / attonitis arcis facies ...), e sembra presupporre una conquista momentanea della rocca capitolina da parte dei Galli $(1,625$... Gallisque ex arce fugatis; 6 , $555 \mathrm{~s}$.... captaeque ... /... arcis $)^{16}$. Tuttavia la stessa voce non ricorda mai il pagamento di un riscatto: dall'unico tangenziale riferimento all'episodio ricaviamo soltanto che la spada gettata da Brenno sulla bilancia ${ }^{17}$ faceva bella mostra di sé fra i trofei di guerra custoditi nella curia del senato di Roma (1, $624 \mathrm{~s}$. hic galeae Senonum pensatique improbus auri / arbiter ensis inest ...).

10 L'imbarazzante dettaglio dell'oro versato dai Romani agli invasori trova, viceversa, spazio nella 'propaganda' di parte opposta. Già a Sagunto, per es., la voce di Annibale si leva verso la nave che porta a Cartagine gli ambasciatori latini, minacciando un nuovo 
(stavolta, fatale per Roma) attacco al Campidoglio: un attacco che non si fermerà davanti all'offerta di un riscatto (2, 33 ss. Tarpeios iterum scopulos praeruptaque saxa / scandatis licet et celsam migretis in arcem, / nullo iam capti vitam pensabitis auro). Quindi, in occasione della battaglia del Ticino, se ne fanno portavoce gli stessi Galli, quelli aggregatisi ad Annibale appena disceso dalle Alpi $(4,143$ ss.) e che, durante la prima parte dello scontro, sottraggono addirittura la ribalta ai Cartaginesi $(4,189 \mathrm{~s}$. nec locus est Tyriis belli pugnaeve, sed omnem / Celticus implevit campum furor...). Il loro capo, Crisso, nella sua follia mitomane si vanta addirittura di discendere da Brenno (4, 150 ipse tumens atavi Brenni se stirpe ferebat) - che pure aveva guidato delle tribù di Senoni, non di Bô̂ -, e imbraccia uno scudo istoriato con la scena della pesatura dell'oro (4, $151 \mathrm{ss}$.... et in titulos Capitolia capta trahebat / Tarpeioque iugo demens et vertice sacro / pensantes aurum Celtas umbone gerebat). Crisso muore di lì a poco, trafitto dalla lancia di Scipione padre, subito dopo aver pronunciato per l'ultima volta il nome dell'antico capo dei Senoni (4, 280 s.): ma fino all'ultimo egli aveva cercato di riattualizzare il passato (o meglio una parte di esso: l'incendio e il saccheggio di Roma), nella vana illusione di riscriverlo in senso filo-gallico (279 ss. "non si è salvato nessuno dalla presa e dall'incendio di Roma, per raccontarti che razza di guerrieri siamo noi, gente di Brenno?..."). La risposta con cui Scipione accompagna il colpo mortale inflitto all'avversario ribadisce con orgoglio nazionalistico l'inviolabilità del Campidoglio (4, 286 ss. ... "ferre haec umbris proavoque memento, / quam procul occumbas Tarpeia sede, tibique / haud licitum sacri Capitolia cernere montis") e finisce per ridimensionare anche 'storicamente' la figura di Crisso, invero una copia alquanto sbiadita del leggendario Brenno ${ }^{18}$.

11 In altre circostanze le immagini esemplari del passato emergono diversamente alla superficie del testo, ovvero non in modo frammentario e dialettico, ma una tantum, sotto forma di parentesi evocative complete e in sé concluse. Si tratta di scorci sulla storia più antica dell'Urbe che risalgono via via sempre più indietro, dall'età arcaica della res publica fino a raggiungere, passando per la Roma dei re, l'epoca leggendaria di Enea ${ }^{19}$. Non è più una storia raffigurata in manufatti, in oggetti artistici (scudi o pitture templari), ma è affidata per intero alla memoria di narratori interni, di personaggiracconto che si trovano occasionalmente, per varie ragioni, vicini ad Annibale (prigionieri di guerra, disertori ecc.). Ora, il fatto che proprio Annibale costituisca l'immediato destinatario di questi racconti comporta una differenza sostanziale rispetto al flash-back sulla vicenda di Regolo del libro VI, dove Serrano incarnava, all'interno del testo, il modello dell'ascoltatore ideale. Collocati entro una sceneggiatura che ne presuppone una prima ricezione 'antagonistica', contraria a quella programmata sul lettore romano che ne trae consolazione e incoraggiamento, questi inserti narrativi sono idealmente chiamati ad arginare, o quanto meno a controbilanciare, la serie dei successi militari di Annibale. Subito dopo vittorie clamorose (in particolare quelle al Trasimeno e a Canne) che sembrano avallarne le aspirazioni di conquista, oppure al momento di concretizzare il suo progetto ossessivo (salire da vincitore sulla rocca del Campidoglio), il condottiero cartaginese si trova puntualmente di fronte - oltre ad intimazioni divine più o meno sollecite (per es. 10, 366 ss.; 12, 701 ss. ecc.) - la sagoma ammonitrice del passato di Roma. Si tratta di un nemico difficile da affrontare con armi convenzionali, un nemico che nella sua nuova veste di racconto, a differenza delle pitture 'provocatorie' del tempio di Literno, non concede neppure l'illusoria sensazione di poter essere cancellato mediante la distruzione materiale. 

cunctator anche sotto il profilo anagrafico e lo assimila all'omerico Nestore che, oltre ad essere sopravvissuto nei secoli a tre generazioni di eroi (ma Fabio è ancora molto più ... energico del vecchio condottiero iliadico) ${ }^{23}$, era stato anche (come Fabio) l'unico superstite di un'antica strage familiare, quella dei figli di Neleo uccisi da Eracle ${ }^{24}$. La presenza di una testimonianza analoga in una nota del commento di Servio a Virgilio (adAen. 6, 845 unus tantum superfuit, Fabius Maximus, qui propter teneram adhuc pueritiam in civitate remanserat. $\underline{\text { Hic }}$ postea cum Hannibalis impetum ferre non posset, mora eum elusit ... et ad Campaniam traxit, ubi deliciis eius virtus obtorpuit ecc.) fa pensare che la notizia possa non essere un'invenzione di Silio, ma risalire magari a qualche escogitazione celebrativa di matrice annalistica. Un indizio in tal senso potrebbe indirettamente celarsi nella stessa ambiguità con cui Livio si era pronunciato sull'unico, giovanissimo, Fabio scampato alla strage soltanto perché lasciato a casa $(2,50,11$... unum propter impuberem aetatem relictum, stirpem genti Fabiae dubiisque rebus populi Romani saepe domi bellique vel maximum futurum auxilium). La narrazione di Cilnio sembra, perciò, riflettere il modus operandi di un'epica storica fortemente impegnata a stabilire una linea di continuità (anche letterale, materiale) tra passato e presente, incline a scoprire analogie fra figure leggendarie e personaggi realmente vissuti che non di rado risultano più 'completi' dei loro stessi modelli, un'epica decisamente proiettata verso l'obiettivo della mitopoiesi, in particolare verso la riesumazione antiquaria di miti del passato. La sapiente e disinvolta manipolazione di fonti, la libertà nel ricomporre i vari aspetti di una tradizione valorizzando rarità documentarie o accostando versioni di un evento tra loro discordanti (e, magari, 'di parte') rappresentano una componente essenziale di tale operazione.

Come il primo, anche il secondo racconto si situa in una fase critica per le sorti di Roma, anzi nel momento forse più angoscioso, quello dell'epilogo tragico della battaglia di Canne. A fungere da cerniera tra due scene retoricamente molto elaborate - la prima, 
in cui il giovane Scipione impedisce la fuga per mare dei fuggitivi guidati da Lucio Cecilio Metello ${ }^{25}$; la seconda, quella del ritrovamento del cadavere di Lucio Emilio Paolo e degli onori funebri tributatigli da Annibale ${ }^{26}$-, Silio Italico inserisce un breve intermezzo legato solo da un tenue filo al contesto narrativo principale (10, 449-502).

Mentre effettua una ricognizione del campo di battaglia ricoperto dai cadaveri dei nemici uccisi (tradendo lo stesso perverso compiacimento che caratterizza il Cesare lucaneo dopo Farsalo) ${ }^{27}$, Annibale rimane colpito da una scena straordinaria e commovente. Un soldato romano, trafitto da innumerevoli dardi, giace a terra in fin di vita esalando nei lamenti il suo ultimo respiro (10, $455 \mathrm{~s}$. extremo ... / murmure). A un certo punto, però, il suo (ormai ex) cavallo ne riconosce la voce e, come rispondendo a una sorta di richiamo, ha una pronta reazione: sbalza di sella il cartaginese Bagèso, il guerriero che nel frattempo si era impossessato di lui (458 ss.), e accorre dal non dimenticato padrone (461 ss.). Con premura affettuosa, l'animale tenta inutilmente di rianimare il soldato; si inginocchia sulle zampe anteriori offrendogli il dorso e invitandolo a riprendere il felice sodalizio (464 ss. inde inclinatus colla et submissus in armos / de more inflexis praebebat scandere terga / cruribus ac proprio quodam trepidabat amore). Clelio - questo il nome del soldato romano - era stato, infatti, un abilissimo cavallerizzo, che assieme al suo destriero prediletto aveva formato una coppia così affiatata da sembrare addirittura in grado di emulare, seppure in una versione vagamente ludica e circense, le gesta del mitico binomio Castore-Cillaro (467 ss.).

Turbato dalla nobiltà 'umana' di quel gesto ${ }^{28}$, Annibale vuole saperne di più (472 ss.). A dargli le informazioni desiderate è Cinna, un disertore che aveva stoltamente preferito confidare nel successo finale dei Cartaginesi ${ }^{29}$. L'atteggiamento del personaggio, contrariamente a quello provocatorio assunto da Cilnio, è assai deferente (478 ductor fortissime). Forse, anzi, lo è fin troppo, dal momento che egli corre il rischio di urtare la suscettibilità del possibile (magari già in un prossimo futuro) padrone di Roma. In effetti, la storia raccontata da Cinna testimonia sì il passato monarchico della città, ma si concentra in particolare sulla fine 'traumatica' di quella forma di governo autoritario (479 ss. ... quondam sub regibus illa, / quae Libycos renuit frenos, sub regibus olim / Roma fuit. Sed enim solium indignata Superbi / ut sceptra exegit ...): all'epoca, insomma, Roma si era già scrollata di dosso (un po' come ha appena fatto il cavallo di Clelio) il peso opprimente del tiranno straniero. Cinna rievoca la vicenda di un'illustre antenata dello sfortunato cavaliere: Clelia, la fanciulla che con un gesto coraggioso compiuto in nome della libertà (un esempio di virtus attiva e non solo passiva come quella di altre celebri eroine romane) si era guadagnata - a dispetto del sesso e dell'età - uno spazio meritato fra i grandi nomi della Roma arcaica. Durante la rievocazione questo narratore interno 'cortigiano' si lascia prendere da un inatteso fervore, forse indotto dalla stessa sproporzione tra l'immagine di una bambina non ancora dodicenne e l'impresa da lei compiuta $^{30}$. Le parole di Cinna sembrano rivelare un'intima tensione. Da un lato, esprimono un'aspirazione alla pace, alla composizione dei conflitti (488 ss. ... mox pace probata / compressere odia, et positum cum foedere bellum; 494 pignora pacis), e arrivano quasi a rammaricarsi dell'indole caratteristica di Romani e Italici, così indomabile e assetata di gloria (490 ss. ... mansuescere corda / nescia, pro superi!, et nil non immite parata / gens Italum pro laude pati). Dall'altro, esse lasciano trapelare un'istintiva ammirazione per il valore della fanciulla, capace di oscurare quello di altri eroi di sesso maschile(496 facta virum sileo... ${ }^{31}$. Con l'ingenuità tipica dei bambini, Clelia compie un atto di ribellione totale (496 s. ... rege haec et foedere et annis / et fluvio spretis; cfr. Sen. ad Marc. 16,2 contempto et hoste et flumine), ed è proprio la raffigurazione di quest'indole pronta a 
osare di tutto anche nelle situazioni più difficili che dovrebbe suonare come monito anche al destinatario interno del racconto, Annibale (499 ss. cui si mutasset sexum natura, reverti / forsan Tyrrhenas tibi non licuisset in oras, Porsena ...). A questo punto, Cinna si accorge di essersi forse spinto un po' troppo oltre e si affretta a concludere accampando ragioni di opportunità (in parte anche letteraria): $501 \mathrm{~s}$.... sed iuveni, ne sim tibi longior, hinc est / et genus et clara memorandum virgine nomen.

Questo brano assolve sicuramente una funzione esemplare nell'economia del racconto principale dove, insieme all'intervento precedente di Scipione e al successivo elogio funebre di Emilio Paolo pronunciato dal condottiero nemico, compone un trittico di scene che mirano a suggerire al lettore il senso della ferma volontà romana di reagire ad ogni costo alla sconfitta subita. D'altra parte, la rievocazione di un così celebre evento del passato, effigiato tra l'altro nello stesso scudo virgiliano di Enea (Aen. 8, 651 et fluvium vinclis innaret Cloelia ruptis), serve - ad un altro livello - all'autore del poema per prendere indirettamente posizione su una questione di antiquaria. Come osserva Ogilvie $^{32}$, le fonti si dividono a più riprese su diversi aspetti della storia di Clelia (la restituzione imposta o volontaria degli ostaggi a Porsenna, la presenza o meno tra loro di Valeria, la figlia di Publicola ecc.), e una simile incertezza grava anche sulle modalità della fuga dell'eroina e delle altre fanciulle dal campo degli Etruschi. In particolare, un filone della tradizione parla di una fuga a cavallo ${ }^{33}$. Ma Silio, a quanto pare sulla scia di Livio, opta senz'altro per la versione secondo cui Clelia sarebbe fuggita a nuoto affrontando le acque di un Tevere 'stupito' (10, $497 \mathrm{s.})$ :

... mirantem interrita Thybrim tranavit frangens undam puerilibus ulnis

19 Il nesso verbale richiama evidentemente il passo di Livio (2, 13, 6 dux agminis virginum inter tela hostium Tiberim tranavit), e anche altrove risulta chiaro che Silio presuppone questa versione (13, 828 illa est, quae Thybrim, quae fregit Lydia bella; cfr. anche Dion.Hal. $5,33,1)$ : la precisazione puerilibus ulnis $(10,498)$ formalizza l'intento di eliminare ogni possibile motivo di dubbio, anche rispetto alle più generiche espressioni impiegate da Livio stesso e Virgilio.

20 Mi pare, tuttavia, che la saldatura della storia di Clelia a quella di un suo pronipote abile cavallerizzo sia implicitamente un modo di ridimensionare o, meglio ancora, di ricollocare (senza rinunciarvi) un elemento che emerge da una pur cospicua tradizione alternativa. La vicenda di Clelio attrae completamente su di sé il tema equestre, che in altri resoconti della storia della sua antenata aveva giocato - come abbiamo appena visto - un ruolo narrativo, addirittura aperto a sviluppi di tipo celebrativo ed eziologico. Mi riferisco alla statua di fanciulla a cavallo che, secondo alcune testimonianze, sarebbe stata eretta in summa Sacra via in onore di Clelia, prima donna a ricevere un simile privilegio ${ }^{34}$. Dunque Silio Italico procede anche a una ricomposizione personale del mosaico della tradizione, secondo un gusto eclettico che - mentre cura il valore esemplare del racconto e la sua funzione interna all'economia dell'opera dimostra ancora l'intenzione di suggerire la continuità del passato, permettendo addirittura a quest'ultimo di trascorrere con naturalezza nel presente della narrazione.

La reazione di Annibale a questo racconto non è precisata: le parole di Cinna sono praticamente interrotte dal clamore suscitato dalla scoperta del cadavere di Emilio Paolo (10, 503 ss.). Il narratore interno, pur correndo a tratti qualche rischio (l'entusiasmo per la virtù di Clelia non era propriamente auspicabile in una strategia 'cortigiana'), non arriva certo a sfidare apertamente l'orgoglio del condottiero, come 
aveva fatto il prigioniero Cilnio nell'excursus su Fabio (7, 69 ss.: Annibale trattiene l'ira al fine di non dare soddisfazione allo sfidante, avidus leti). Per il momento Annibale incassa con notevole disinvoltura la lezione ammonitrice del passato. L'orgoglio per il successo appena conseguito gli ispira perfino un'accorta condotta autocelebrativa: mentre elogia il valore del console defunto, egli esalta indirettamente le proprie qualità di condottiero.

In seguito, però, le circostanze evolvono in maniera contraria alle sue attese: l'esercito cartaginese rimane vittima dell'atmosfera corrotta di Capua e gli dèi manifestano un ostilità sempre più aperta. Il peso della fama e della storia di Roma torna perciò a contrastare Annibale in modo più concreto, al punto da riuscire a dissuaderlo dal proseguire nella lotta. Anche un destinatario così indifferente o ribelle finisce per essere costretto ad arretrare davanti alla potenza soverchiante di queste 'simbiosi cronologiche' tra presente e passato. In occasione dell'attacco portato alle mura di Roma (libro 12), Annibale non prende la decisione di ritirarsi definitivamente neppure dopo le intimazioni di Giunone (701 ss.), che gli svela la ragione dei suoi tentativi infruttuosi e gli presenta gli dèi olimpî intenti ognuno a combatterlo dal proprio colle (706 ss.). Egli cede soltanto dopo aver ascoltato (e questa volta senza averlo richiesto!) un ultimo racconto, la cui protagonista è Minerva. Questa divinità, tra l'altro particolarmente onorata dai Flavi e soprattutto da Domiziano, possiede uno statuto abbastanza ambiguo nei Punica: a Canne, per es., aveva combattuto attivamente dalla parte dei Cartaginesi in omaggio alla sua nascita africana (presso il libico lago Tritone, da cui l'epiteto di Tritonia), e in memoria della sua antica milizia antitroiana (9, 297; 439 e 460 ss.). Alla fine della battaglia, richiamata all'ordine da Giove, ella si sforza di giustificare quello che era apparso, piuttosto, un comportamento antiromano: la dea afferma di aver voluto salvare la vita di Annibale per consentirgli di portare a termine una grande impresa (9, 530 ss. quamquam ego non Teucros (nostro cum pignore regnet / Roma et Palladio sedes hac urbe locarim) / non Teucros delere aderam, sed lumen alumnae / Hannibalem Libyae pelli florentibus annis / vita atque exstingui primordia tanta negabam). Successivamente, tuttavia, pur senza impegnarsi in prima persona (così come Giunone) nella battaglia in difesa di Roma, Minerva vi gioca un ruolo indiretto e determinante proprio attraverso il racconto di un evento del passato, con cui si abbandona la prospettiva storica (ancorché ammantata di leggenda) dei casi precedenti per risalire più indietro ed entrare senza mezzi termini nella sfera del mito.

L'estremo impulso titanico del condottiero cartaginese, ormai non più assecondato neppure dall'entusiasmo dei soldati, si infrange davanti alle parole di un certo Dasio di Argyripa/Arpi, il quale dopo la disfatta di Canne aveva scelto di passare dalla parte dei nemici di Roma $(13,30 \text { ss.) })^{35}$. L'introduzione di questo personaggio, altrove non menzionato dalle fonti, potrebbe essere stata suggerita a Silio dalla frequenza di figure omonime nel testo di Livio, tutte ugualmente ostili alla causa romana. Lo storico di età augustea racconta per es. che, dopo Canne, un certo Dasio Altinio avrebbe consegnato Arpi ai Cartaginesi, cercando quindi, in un secondo tempo, di ricondurla ai Romani (Liv. 24, 45, 1 ss.); in precedenza un altro Dasio, proveniente da Brindisi e comandante del presidio di Clastidium, aveva 'venduto' la città ad Annibale dopo la battaglia del Ticino (Liv. 21, 48, 9); più oltre (Liv. 26, 38, 6) troviamo, infine, menzionato un terzo Dasio, capo di Salapia e amico di Annibale.

Dimostrando una spiccata e niente affatto gratuita inclinazione antiquaria, il Dasio di Silio Italico va addirittura a ripescare nella memoria alcune storie che il suo celebre 
antenato, l'eroe greco Diomede, rifugiatosi in Apulia al termine di molte traversie, era stato spesso invitato a ripetere alla tavola dell'ospite Dauno. Argomento dei racconti, cuciti insieme da Dasio per l'occasione, sono il ratto del Palladio, che permise ai Greci di conquistare Troia $(13,36-50)$, e la successiva espiazione dell'impresa, ovvero la riconsegna del simulacro da parte dello stesso Diomede a Enea giunto nel Lazio (13, 51-81). È proprio in questa seconda parte della storia che si concentra in particolare il messaggio rivolto al destinatario interno, Annibale, per indurlo a riflettere sull'inopportunità di proseguire l'assedio di Roma ${ }^{36}$. Si tratta di una delle varianti più rare della vicenda, che ci è attestata indirettamente da una fonte annalistica (C. Cassio Hemina 1, 97, 7 Peter [ap. Solin. 2, 14]) ed è riferita più volte, con alcune oscillazioni, anche da Servio, da cui viene fatta risalire a Varrone (ad Aen. 2, 166; 3, 407 e 550; 5, 81; l'opera di Varrone potrebbe essere il De familiis Troianis, cfr. Serv. ad Aen. 5, 704). Secondo Cassio Hemina, Enea si trovava già nel Lazio (in agro Laurenti posuisse castra) ${ }^{37} \mathrm{e}$ stava dedicando alla madre Venere una statua che aveva portato con sé dalla Sicilia (dum simulacrum, quod secum ex Sicilia advexerat, dedicat Veneri matri, quae Frutis dicitur) ${ }^{38}$, quando ricevette la visita di Diomede che gli riconsegnò il Palladio (a Diomede Palladium suscepit). Un'analoga collocazione dell'episodio nel Lazio è presupposta anche dall'Origo gentis Romanae (12), dove però l'incontro avviene tra Enea e Ulisse (cfr. sotto). Da parte sua, Servio (sulla base di Varrone) narra che Diomede, tormentato da ansie e pericoli dopo il furto del Palladio, fu indotto dal responso di un oracolo a restituirlo al capo dei profughi troiani ${ }^{39}$. Con questa intenzione egli si fece incontro ad Enea appena approdato sulle coste della Puglia per effettuare una sosta ${ }^{40}$ : ma quest'ultimo, impegnato a compiere un sacrificio augurale, evitò di rivolgere lo sguardo verso di lui per non violare il rito, e così fu un certo Naute a ricevere la statua da Diomede (Serv. ad Aen. 2, 166 e 3, 407) $)^{41}$. Secondo Servio, Virgilio alluderebbe tacitamente all'episodio quando racconta la sosta calabra presso castrum Minervae (Verg. Aen. 3, 531 iam propior templumque apparet in arce Minervae, e 543 s. tum numina sancta precamur / Palladis armisonae, quae prima accepit ovantis). Il recupero di un'eziologia così preziosa non è comunque indizio di passione antiquaria fine a se stessa: Silio dimostra anche in questo caso, come vedremo subito, di saper scegliere nel repertorio della tradizione la versione più adatta alle esigenze del contesto narrativo principale, sceneggiando di fronte all'indomito Annibale l'esemplare atto di sottomissione (davanti al passato e al presente) di un grande eroe omerico.

All'arrivo in Apulia, dunque, Diomede aveva fondato una città e l'aveva dotata di un tempio in onore di Minerva, dove la preziosa statua avrebbe dovuto trovare una degna collocazione (Sil. 13, 51 ss.). Ma intanto egli era sempre più assillato da rimorsi e angosce (52 aeger delicti) ${ }^{42}$ e i tentativi di scacciare i fantasmi del passato si rivelavano inutili. Questa caratterizzazione di Diomede, della sua ansia di pace e di espiazione nei confronti della dea 'frigia' (52 s. Phrygium ... / numen) ${ }^{43}$ e dei Penati di Troia, ricalca ampiamente quella dell'XI libro dell'Eneide (243 ss.) e, sotto certi aspetti, ne drammatizza il patetismo. In Virgilio l'ambasciatore dei Rutuli Venulo riferiva a Turno le parole con cui l'eroe greco gli aveva rievocato le sofferenza della guerra (Aen. 11, 252 ss.) e, quindi, i tragici ritorni di molti capi greci colpiti dall'ira di Minerva (259 ss. ... scit triste Minervae / sidus ...), finendo col dichiarare solennemente la propria intenzione di non voler più combattere contro i Troiani $(277$ ss.) e, anzi, invitando i Rutuli a portare doni a Enea anziché a lui (281 s. munera, quae patriis ad me portatis ab oris, / vertite ad Aenean...). 
asi ricollegandosi alle parole 'riferite' del Diomede virgiliano ${ }^{44}$, Dasio narra che una notte all'eroe apparve in sogno proprio l'immagine di una Minerva adirata: questa lo rimproverò di aver scelto per lei una sede oscura e periferica come la terra di Dauno e lo esortò a cercarle una sistemazione più degna presso coloro che la ospitavano in precedenza, attuali abitatori del Lazio e fondatori di una 'Troia migliore' (Sil. 13, 58 ss.):

non haec, Tydide, tantae pro laudis honore

digna paras; non Garganus nec Daunia tellus

debentur nobis. quaere in Laurentibus arvis

qui nunc prima locant melioris moenia Troiae.

huc vittas castumque refer penetrale parentum.

Prima ancora che sull'epifania del dio Tevere a Enea nel libro VIII dell'Eneide (26 ss.) ${ }^{45}$, questa scena mi sembra modellata esplicitamente sull'apparizione notturna e le raccomandazioni rivolte, sempre ad Enea, dai Penati di Troia durante la sosta nell'isola di Creta (Verg. Aen. 3, 154 ss.):

(159 ss.) ... tu moenia magnis

magna para longumque fugae ne linque laborem.

mutandae sedes. Non haec tibi litora suasit

Delius ...

(163) est locus, Hesperiam Grai cognomine dicunt ...,

(167) hae nobis propriae sedes; hinc Dardanus ortus ...

Si tratta, ovviamente, di un modello contrastivo. In Virgilio Enea riceve un'indicazione positiva dagli dèi di cui è detentore e custode: i Penati di Troia correggono il suo errore d'interpretazione e lo confortano (Aen. 3, 153 curas ... demere) mostrandogli la giusta direzione; non intendono affatto abbandonarlo, come viceversa è il caso della Minerva di Silio Italico, minacciosa (13, 57 minitans) e soprattutto stanca di risiedere presso lo 'straniero' Diomede. Proprio il tono autoritario e assai poco solidale con cui la dea manifesta la volontà di romanizzarsi, abbandonando il precedente luogo di culto e soprattutto annunciando l'inevitabile separazione dall'attuale tutore, ricorda da vicino il passo del libro IV dei Fasti di Ovidio in cui Cibele (lei sì Phrygium numen per antonomasia) ${ }^{46}$ dichiarava a un Attalo perplesso l'intenzione di seguire gli ambasciatori romani intenzionati a trasferirne il culto da Pergamo a Roma (fast. 4, 269 s.):

ipsa peti volui, nec sit mora, mitte volentem.

dignus Roma locus, quo deus omnis eat ${ }^{47}$

Alla luce di questo confronto, la scena del libro XIII dei Punica costituirebbe una piccola anticipazione della più ampia e articolata rilettura che del racconto dei Fasti Silio farà successivamente, nell'ultima parte del poema, al momento di narrare la traslazione del culto della Grande Madre a Roma e la storia di Claudia Quinta $(17,1-47)^{48}$. Nel racconto di Dasio, tra l'altro, l'intenzione di far reagire insieme due modelli così strettamente collegati fra di loro (gli episodi di Aen. 3 e fast. 4) è confermata da alcuni dettagli complementari. Già il modo in cui il disertore si accinge ad iniziare il racconto $(13,35$ is volvens veterum memorata antiqua parentum) richiama la movenza introduttiva delle parole di Anchise in Aen. 3, 102 tum genitor veterum volvens monimenta virorum. Proprio Anchise, con la sua errata interpretazione dell'oracolo di Apollo Delio (Aen. 3, 96 antiquam exquirite matrem), sta per indirizzare i profughi troiani verso Creta, la patria di... Cibele (111 hinc mater cultrix Cybeli ...), ovvero la dea materialmente 'evocata' nel brano dei Fasti. Così, non appare strano che il Diomede di Silio riceva l'ammonimento onirico di Minerva mentre sta fondando la nuova città e il tempio della dea: prima del sogno rivelatore, a Creta, anche Enea si stava adoperando a far nascere una nuova 
Pergamo (Aen. 3, 132 ss. ergo avidus muros optatae molior urbis / Pergameamque voco...; 137 iura domosque dabam ...).

D'altro canto, Ovidio costruisce esplicitamente l'episodio del libro IV dei Fasti in risposta a quello virgiliano. Questa volta il responso dell'oracolo di Apollo non lascia spazio a dubbi, almeno per quanto riguarda l'obiettivo principale, che è indiscutibilmente Cibele, una 'madre' da scomodare e far venire dalla sua sede, non più da raggiungere (fast. 4, 263 divum ... arcessite matrem) ${ }^{49}$. I Romani non corrono più il rischio di interpretare erroneamente la lieve ambiguità di Apollo (fast. 4, 264 ... in Idaeo est invenienda iugo), e andare magari a cercare la dea a Creta ripetendo l'errore di Enea fuorviato da Anchise (Verg. Aen. 3, 104 s. Creta Iovis magni medio iacet insula ponto, / mons Idaeus ubi et gentis cunabula nostrae). Una volta giunti a Pergamo, gli ambasciatori trovano una divinità assai compiacente, che dichiara la sua ferma volontà di seguirli in Italia: cosa che, del resto, avrebbe voluto già fare (se i tempi fossero stati maturi) al momento della partenza dell'esule Enea (fast. 4, 251 s. cum Troiam Aeneas Italos portaret in agros, / est dea sacriferas paene secuta rates).

Ma torniamo a Silio Italico. Terrorizzato dal prodigio (come l'Attalo di fast. 4, 271 ... soni terrore pavens), Diomede parte subito alla volta del Lazio al fine di restituire il Palladio a Enea e alla nuova città che egli sta già fondando dopo aver sconfitto i Latini e aver sposato Lavinia (Sil. 13, 64 s. iam Phryx condebat Lavinia Pergama victor ...; e Phryx in relazione ad Enea compare proprio in fast. 4, 274 Phryx pius) ${ }^{50}$. Quando riconoscono davanti alla loro città l'accampamento del grande e temuto avversario di un tempo (Sil. 13, 67 castra) i Troiani paventano un ritorno del passato, il compimento di timori già espressi da Venere a Giove nel X dell'Eneide (28 s. atque iterum in Teucros Aetolis surget ab Arpis / Tydides ...) $)^{51}$. Ma, proprio come nell'Eneide (cfr. l'invito agli ambasciatori rutuli di 11, 292 coeant in foedera dextrae; e già 278 ne vero, ne me ad tales impellite pugnas), Diomede non è neppure sfiorato dall'idea di ricominciare a combattere. Anzi, il suo atteggiamento risulta subito decisamente conciliante ${ }^{52}$, e si dice pronto ad accettare un'alleanza pacifica (Sil. 13, 76 dextras iungamus inermes). A dimostrazione della sua buona fede, l'eroe esibisce davanti ai Troiani spaventati e diffidenti il vero e unico simbolo della presenza protettiva di Minerva, il Palladio (13, $77 \mathrm{~s}$.... veniamque precatus / Troianam ostentat trepidis de puppe Minervam) ${ }^{53}$. L'espiazione del misfatto compiuto si oggettiva in un gesto che per certi versi suona quale implicita riparazione del perfido 'dono' del traditore Sinone (Verg. Aen. 2, 162 ss.). Il racconto di Sinone, un narratore inaffidabile, partiva proprio dal ratto del Palladio, che veniva capziosamente presentato come la causa di tutte le sventure dei Greci, e non come l'impresa fatale tesa a provocare la futura caduta di Troia: i 'ritorni' degli Achei, narrati da Diomede in Aen. 11, 255 ss., avrebbero manifestato che in quelle parole si celava, in fondo, un barlume di verità.

Per dimostrare ad Annibale la necessità di rinunciare al suo progetto, Dasio (evidentemente abile, come almeno due dei suoi omonimi liviani, nel cogliere le alterne fortune della guerra e adeguarvisi di buon grado) conclude il racconto con l'aition dell'inespugnabilità di Roma, senza dimenticare di fornirne le prove 'storiche' (la sconfitta dei Galli: 13,79 ss.):

haec ausos Celtas irrumpere moenia Romae corripuit leto neque tot de milibus unum ingentis populi patrias dimisit ad aras. 
attribuzione a Minerva di un ruolo decisivo nella vittoriosa resistenza all'assedio gallico non trova riscontri ulteriori ${ }^{54}$. Essa potrebbe, a sua volta, risentire di una tradizione relativa all'impegno profuso nella stessa circostanza da Vesta, la dea che ospita concretamente nel suo penetrale i pignora imperii di Roma, i Penati e con ogni probabilità anche il Palladio ${ }^{55}$. due divinità. Subito dopo l'aition dell'altare dedicato a Iuppiter pistor sul Campidoglio (un aition che risale appunto all'epoca dell'assedio gallico, quando Vesta svolse attivamente il suo compito di divinità protettrice di Roma), il poeta elegiaco rievoca la storia dell'origine del Palladio, pegno della sopravvivenza di Troia, e delle sue vicissitudini posteriori (fast. 6, 419 ss.). Ovidio infrange giocosamente la tradizione (recepita, come abbiamo visto, nell'Eneide) ${ }^{56}$ secondo la quale Minerva avrebbe reagito con violenza alla propria 'deportazione' da Troia: ancora indispettita per l'esito del giudizio di Paride, la dea addirittura favorisce il suo stesso rapimento e la conseguente caduta della città (fast. 6, 431 s. sub Priamo servata parum: sic ipsa volebat, / ex quo iudicio forma revicta sua est). Ma al narratore dei Fasti non interessa tanto mettere ordine in controverse questioni di antiquaria, ovvero stabilire chi abbia rubato il Palladio da Troia (fast. 6, 433 s. seu genus Adrasti, seu furtis aptus Ulixes, / seu fuit Aeneas, eripuisse ferunt). Ciò che davvero conta, in definitiva, è che il Palladio sia arrivato a Roma: Minerva è res Romana, e Vesta ne è l'assoluta garante e custode (435 s. auctor in incerto, res est Romana, tuetur / Vesta quod assiduo lumine cuncta videt), così come il Palladio stesso lo è della grandezza eterna di Roma.

La rassegnazione con cui il Diomede di Silio Italico accetta il destino (e la volontà di una dea 'amica' come Pallade) è proposta davanti ad Annibale come un modello di comportamento e questa volta, finalmente, l'esempio produce l'effetto auspicato. Turbato dalle parole di Dasio, il condottiero cartaginese ordina la ritirata definitiva (13, $82 \mathrm{~s}$. his fractus ductor convelli signa maniplis / optato laetis iubet): il suo sogno di salire sul Campidoglio, e di detronizzare Giove, è definitivamente tramontato ${ }^{57}$.

Nella sceneggiatura dei Punica tale risultato è ottenuto grazie ad uno sforzo collettivo, che ha visto impegnati insieme uomini e dèi; uno sforzo al cui felice esito ha contribuito in modo senza dubbio determinante anche il passato leggendario di Roma, 'combattendo' Annibale in circostanze cruciali e vanificandone le ambizioni (fondate, a loro volta, su una lettura antagonistica del passato, sull'appropriazione propagandistica delle precedenti sconfitte romane). Questa vitalità immanente e pervasiva della tradizione, che al momento opportuno emerge a suggerire comportamenti e ad illustrarli efficacemente con esempi (talora 'viventi'), rappresenta un aspetto non secondario della potenza di Roma. D'altra parte lo stesso fenomeno assume anche precise implicazioni di tipo letterario nel poema di Silio Italico, che nella costante ed esibita tensione a 'riattualizzare' le opere della grande stagione augustea, e a ricostruire così il proprio passato genealogico, trova la sua principale ragion d'essere. 


\section{BIBLIOGRAFIA}

Ahl-Davis-Pomeroy 1986: F.M. Ahl - M.A. Davis - A. Pomeroy, Silius Italicus, in ANRW II 32.4 (1986) 2492-2561.

von Albrecht 1964: M. von Albrecht, Silius Italicus. Freiheit und Gebundenheit römischer Epik, Amsterdam 1964.

von Albrecht 1968: M. von Albrecht, Claudia Quinta bei Silius Italicus und bei Ovid, “AU” 11 (1968), pp. 76-95.

Barchiesi 2001: A. Barchiesi, Genealogie letterarie nell' epica imperiale : fondamentalismo e ironia, in E.A. Schmidt (ed.), L'histoire littéraire immanente dans la poésie latine (Entretiens de la Fondation Hardt), Vandœuvres-Genève 2001, pp. 315-354.

Brouwers 1982: J.H. Brouwers, Zur Lucan-Imitation bei Silius Italicus, in AA.VV., Actus. Studies in honour of H.L.W. Nelson, Utrecht 1982, pp. 73-87.

Fantham 1998: Ovid. Fasti. Book IV, comm. by E. Fantham, Cambridge 1998.

Fowler 1996: D. Fowler, Even better than the real thing: a tale of two cities, in J. Elsner (ed.), Art and Text in Roman Culture, Cambridge 1996, pp. 57-74 e 287-293.

Fucecchi 1999: M. Fucecchi, La vigilia di Canne nei Punica e un contributo allo studio dei rapporti fra Silio Italico e Lucano, in P. Esposito - L. Nicastri (edd.), Interpretare Lucano, Univ. di Salerno. Quaderni del Dip. di Scienze dell'Antichità, Napoli 1999, pp. 305-342.

Fucecchi 2003: M. Fucecchi, I Punica e altre storie di Roma nell'epos di Silio Italico, in A. Casanova - P. Desideri (edd.), Evento, racconto, scrittura nell'antichità classica. Atti del Convegno Internazionale di Studi, Firenze 25-26/11/2002, Univ. di Firenze, Dip. di Scienze dell'Antichità "G. Pasquali", Firenze 2003, pp. 269-292.

Horsfall 2003: Virgil, Aeneid 11. A Commentary by Nicholas Horsfall, Leiden-Boston, Brill 2003.

Mc Guire 1995: D.T. Mc Guire, History compressed: the Roman names of Silius'Cannae episode, "Latomus" 54 (1995) 110-118.

Meyer 1924: K. Meyer, Silius und Lucan, Diss. Würzburg 1924.

Niemann 1975: K.H. Niemann, Die Darstellung der römischen Niederlagen in den Punica des Silius Italicus, Bonn 1975.

Ogilvie 1965: R.M. Ogilvie, A commentary on Livy, Books I-V. Oxford 1965.

Ripoll 2001: F. Ripoll, La restitution du Palladium à Enée chez Silius Italicus(Punica,XIII, 30-81), “Etud. Class." 69 (2001) 353-368.

Santini 1983: C. Santini, La cognizione del passato in Silio Italico, Roma 1983.

Spaltenstein 1986: F. Spaltenstein, Commentaire des Punica de Silius Italicus: livres I-VIII, Genève 1986.

Spaltenstein 1990: F. Spaltenstein, Commentaire des Punica de Silius Italicus : livres IX-XVII, Genève 1990.

Vessey 1975: D.W.T.C. Vessey, Silius Italicus. The shield of Hannibal, “AJPh” 96 (1975) pp. 391-405. 


\section{NOTE}

1. Il ritorno a Roma di Varrone, il console fuggito dal campo di battaglia poco prima della disfatta, è descritto da Silio alla fine del libro X (605 ss.). In Livio la stessa scena chiude la narrazione dell'episodio di Canne (e il libro XXII: 22, 61, 14 s.): lo storico augusteo ha, infatti, concentrato gli avvenimenti precedenti nei due libri iniziali della terza decade.

2. Quelle ad opera dei Cartaginesi, appunto, ma non solo. Già in occasione della battaglia del Ticino, per es., la notizia di truppe galliche attivamente impegnate al fianco di Annibale offre lo spunto per rievocare un incubo del passato: la presa di Roma da parte dei Senoni (cfr. più oltre).

3. Questa istanza trova un'espressione significativa nell'apostrofe che il narratore (collocato nel futuro 'imperiale') indirizza idealmente a Roma, subito prima del tragico resoconto di Canne: 9 , 349 ss. ... tuque anxia fati / pone, precor, lacrimas et adora vulnera laudes / perpetuas paritura tibi. nam tempore, Roma, / nullo maior eris. mox sic labere secundis, / ut sola cladum tuearis nomina fama. Cfr. von Albrecht 1964 e Niemann 1975.

4. Come nel caso della battaglia contro il fiume Trebbia di 4, 638 ss., una riedizione dell'omerica

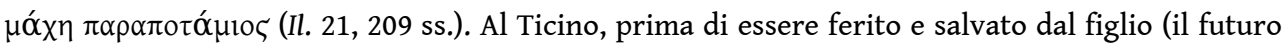
Africano, all'epoca ancora un ragazzino), Scipione aveva ucciso Crisso, il capo dei Galli (cfr. sotto). 5. Flaminio è raffigurato mentre soccombe, da solo, all'assalto dei Galli guidati da un certo Ducario $(5,644$ ss.), desideroso di vendicare la sconfitta inflitta dal console romano ai Boî nel 223 a. C. (cfr. 4, 704 ss.).

6. Per es. la fine che attende Annibale (2, 701 ss., quindi 13, 784 ss.); la vittoria di Scipione Emiliano nella terza guerra punica $(17,374$ s., ma cfr. già 7,492 s., all'interno della profezia di Proteo).

7. Le anticipazioni del futuro più lontano non sono, in verità, sempre liete: accanto alla profezia di Giove $(3,571$ ss.), che annuncia la vittoria finale nella guerra e si spinge fino a pronosticare l'avvento della dinastia dei Flavî, si profila l'incubo minaccioso delle guerre civili (13, 850 ss.: durante la catabasi, la Sibilla presenta a Scipione le anime di Mario, Silla, Pompeo e Cesare). È proprio sulla base di questa consapevolezza che la voce del narratore 'imperiale' assume, talvolta, toni pessimistici che ricordano la maniera di Lucano (per es. nell'elogio della virtù 'sofferente' esibita da Roma a Canne:10, 657 haec tum Roma fuit: post te cui vertere mores / si stabat fatis, potius, Carthago, maneres). L'effetto chiaroscurale prodotto da anticipazioni e commenti pessimistici sul futuro è stato già rilevato da Ahl-Davis-Pomeroy 1986, 2507 ss. Sul rapporto fra i Punica e il Bellum civile di Lucano, cfr. anche Meyer 1924; Brouwers 1982 e, da ultimo, Fucecchi 1999.

8. Di questo aspetto mi sono occupato in un recente contributo di cui il presente lavoro costituisce la naturale prosecuzione (Fucecchi 2003, part. 272 ss.).

9. Su questa ekphrasis, in generale, cfr. già Vessey 1975.

10. Fowler 1996, 57 ss., in part. 63 ss.

11. Nessun cenno alla prigionia e al supplizio patiti in Africa (su cui, peraltro, ampi ragguagli erano stati dati pochi versi prima dal racconto esemplare di Maro): al contrario, secondo una precisa strategia di surenchère, vediamo rappresentata la punizione di Santippo, il mercenario spartano che - dopo aver contribuito con l'inganno a fare prigioniero Regolo - era caduto in disgrazia presso i suoi stessi 'committenti' cartaginesi (Fucecchi 2003, 282 s.).

12. Una probabile esagerazione propagandistica che non trova altri riscontri (Fucecchi 2003, 283 s.).

13. 6,700 ss., e in particolare, per l'auspicio sovversivo della vittoria su Giove Capitolino, 711 ss. haec mitte in populos, et adhuc maiora dabuntur. / flagrantem effinges facibus, Carthago, Libyssis / Romam et deiectum Tarpeia rupe Tonantem (Fowler 1996, 72 s.).

14. Ogilvie 1965, 734, ad Liv. 5, 47.

15. Ogilvie 1965, 736 ad Liv. 5, 48-50 segnala che in altre fonti le cose vanno diversamente: Polibio $(2,18,3)$ sembra presupporre il pagamento del riscatto ma non si sofferma troppo sulla 
questione; secondo Diodoro (14, 117, 7, prob. sulla base di Timeo) i Galli furono subito dopo sconfitti dagli abitanti di Caere in Sabina, che recuperarono il riscatto (cfr. anche Strab. 5, 220; per la sostituzione dei Romani ai Ceretani, cfr. Suet. Tib. 3, 2). Altrove ancora è lo stesso Camillo a recuperare il riscatto già pagato (presso Veascium secondo Diod. 14, 117, 5; presso Pesaro secondo Serv. ad. Aen. 6, 825; cfr. Polyb. 2, 22). La fonte di Livio, secondo la quale Camillo avrebbe 'interrotto' il pagamento giungendo proprio mentre si procedeva a pesare l'oro, potrebbe essere l'annalista Claudio Quadrigario: Ogilvie (in nota a Liv. 5, 48, 8) avanza quest'ipotesi sulla base dell'identità del funzionario romano preposto alla pesatura, Q. Sulpicio (secondo Festo 510 L., probabile testimone di una tradizione antecedente, il personaggio si sarebbe chiamato Appio Claudio). È tuttavia da segnalare che lo stesso Livio, in altri luoghi dell'opera, propone una versione differente: per es. 10, 16, 6 habere accolas Gallos, inter ferrum et arma natos, feroces cum suopte ingenio tum aduersus Romanum populum, quem captum a se auroque redemptum, haud uana iactantes, memorent e 22, 59, 7 maiores quoque acceperamus se a Gallis auro redemisse (portavoce di quest'ultima versione, chiaramente capziosa, è il rappresentante dei soldati romani fatti prigionieri a Canne, che sta cercando di convincere il senato ad attivarsi per favorire la loro liberazione).

16. Ogilvie 1965, 720 ad Liv. 5, 39-43, 5 (cfr. per es. Enn. ann. 227 Sk.; contra Liv. 5, 47 e Spaltenstein 1986 ad Sil. 1, 625).

17. Liv. 5, 48, 9 additus ab insolente Gallo ponderi gladius. Plutarco (Cam. 28, 6) e Zonaras (7, 23) parlano anche di una cintura posta sulla bilancia da Brenno. Dionigi di Alicanasso $(13,9,1)$ aggiungeva il fodero della spada.

18. In questo senso - come mi suggerisce l'anonimo referee della rivista, che ringrazio del contrbuto - il gesto con cui Scipione vanifica i sogni di gloria di Crisso può essere accostato alla pronta reazione di Ascanio alle provocazioni arroganti di Numano Remulo (Verg. Aen. 9, 590 ss., cfr. in part. 634 s. ... i, verbis virtutem inlude superbis ! / bis capti Phryges haec Rutulis responsa remittunt). Col passato, insomma, non si scherza e, come Numano, anche Crisso viene analogamente punito nella sua ingenua presunzione. A sostegno di questa ipotesi si aggiunge indirettamente il fatto che, di lì a poco (4, 454 ss.), il modello del IX dell'Eneide viene esplicitamente attivato in occasione della celebrazione della prima impresa eroica del giovane figlio del console, il futuro Africano (cfr. in part. l'elogio di Marte: 4, 472 ss. , in relazione a quello di Apollo: Verg. Aen. 9, 641 ss.).

19. Questo procedimento è segnalato da Ripoll 2001 (part. 354 s. e 366) che affronta, peraltro, il tema da un punto di vista lievemente diverso dal mio e comunque concentra la sua analisi sull'ultimo dei tre casi (la restituzione del Palladium a Enea da parte di Diomede, su cui cfr. più oltre).

20. Mi limito qui a richiamare i punti principali dell'analisi svolta in Fucecchi 2003, 286 ss.

21. Il personaggio era stato già presentato diffusamente nel libro VI (609 ss.: Giove ispira la sua nomina a dittatore; 619 ss.: esaltazione della sua capacità di ottenere successi in battaglia preservando l'incolumità dei propri soldati; 627 ss.: la genealogia mitica della gens Fabia, discendente dall'unione di Ercole con una figlia dell'arcade Evandro).

22. Cfr. per es. Ov. fast. 2, 237 ss. ut tamen Herculeae superessent semina gentis / credibile est ipsos consuluisse deos. / Nam puer impubes et adhuc non utilis armis / unus de Fabia gente relictus erat; / scilicet ut posses olim te, Maxime, nasci / cui res cunctando restituenda foret.

23. 7, 592 ss. ... maiorem surgere in arma / maioremque dedit cerni Tirynthius. Altae / scintillant cristae et, mirum, velocibus ingens / per subitum membris venit vigor; ingerit hastas / adversumque premit telorum nubibus hostem, / qualis post iuvenem nondum subeunte senecta / rector erat Pylius bellis aetate secunda. Sulle venature paradossali che assume, talora, la valorizzazione encomiastica dell'energia del pur anziano Fabio Massimo (come la già citata espressione certaverit unus ter centum dextris di 7, 63 s., che ne fa una specie di centimane 'buono'), cfr. Fucecchi 2003, 288 e n. 42. 
24. Hom. Il. 11, 692 s.; Ov. met. 12, 542 ss., part. 553 ss. e Apollod. 2, 142.

25. 10, 415-48; cfr. Liv. 22, 53, 4 ss.

26. Solo un accenno in Liv. 22, 52 .

27. 10, $450 \mathrm{~s}$. lustrabat campos et saevae tristia dextrae / facta recensebat ... e 453 dulcia praebebat trucibus spectacula Poenis; cfr. Luc. 7, 786 ss., in part. 794 s. ... iuvat Emathiam non cernere terram / et lustrare oculis campos sub caede latentes e 796 ... laeta ... scelerum spectacula.

28. Un'estremizzazione dell'antico topos della fedeltà dell'animale al cavaliere, in cui è forse da intravedere una risposta 'ortodossa' ad alcune riletture 'anticonformistiche' dello stesso motivo effettuate in età flavia (penso, in particolare a Val. Fl. 6, 203 ss., dove un guerriero scita accusa il cavallo del fratello ucciso di aver accettato troppo supinamente la monta del nemico, il greco Castore). Sull'applicazione di concetti quali 'ortodossia' e 'fondamentalismo' all'epica flavia, unitamente a risvolti di 'ironia' paradossale, cfr. Barchiesi 2001.

29. Il commento sprezzante del narratore onnisciente lo definisce lapidariamente credulus adversis, al v. 477. Il nome del personaggio, che anticipa quello di un futuro protagonista delle guerre civili, non è forse (pace Spaltenstein $1990 \mathrm{ad}$ l.) scelto a caso: cfr. Mc Guire 1995, $110 \mathrm{ss}$.

30. La precisazione inedita dell'età della piccola Clelia (10, $492 \mathrm{~s}$... bis Cloelia senos / nondum complerat primaevi corporis annos) è interessante e curiosa: la stessa età aveva Elena, secondo l'Epitome di Apollodoro (1, 23), quando fu rapita da Teseo; contra, cfr. Diod. 4, 63, 2 dove si dice che aveva 10 anni.

31. Queste parole sembrano alludere implicitamente alle gesta coeve di Muzio Scevola e Orazio Coclite (484), che in Silio non godono del privilegio dell'evocazione narrativa ma solo di più fugaci menzioni 'catalogiche' (a 8, 383 ss. e 13, 726 ss.: sulla superiorità dell'impresa di Clelia, cfr. Liv. 2, 13, 8). Il personaggio di Clelia figura anche nella galleria oltremondana di donne celebri (13, 828 ss.).

32. 1965 ad Liv. 2, 13, 6 ss.

33. Val. Max. 3, 2, 2; Flor. 1, 10 e De vir.ill. 13, 1; cfr. anche Plut. Publ. 19.

34. Liv. 2, 13, 11; cfr. Sen. ad Marc. 16, 2; Plut. Publ. 19; De vir.ill. 13, 4. Meno chiaro Dion.Hal. 5, 35, 2, ma sia Dionigi che Plutarco riferiscono anche che Porsenna, ammirando il valore di Clelia, le fece dono di un cavallo.

35. Su questo episodio cfr. Ripoll 2001, alla cui analisi mi richiamerò nei punti in cui essa integra la presente trattazione.

36. Sulla funzione che il personaggio omerico di Diomede aveva già rivestito nella 'propaganda' anti-troiana e anti-romana di Annibale (e sul modo in cui il racconto di Dasio vi si contrappone dall'interno), svolge alcune giuste osservazioni Ripoll 2001, $355 \mathrm{~s}$.

37. Cfr. Sil. 13, 64 iam Phryx condebat Lavinia Pergama victor (Ripoll 2001, 357).

38. Cfr. Serv. adAen. 1, 720 Venus Erycina, quam Aeneas secum advexit. L'importazione storica del culto di Venere Ericina a Roma, avvenne nel 217 a. C., poco dopo l'inizio della seconda guerra punica.

39. Serv. ad Aen. 2, 166 credens sibi non esse aptum [scil. Palladium] propter sua pericula, quibus numquam cariturum responsis cognoverat , nisi Troianis Palladium reddidisset... (il carattere tondo evidenzia le aggiunte del Danielino). Ripoll 2001, 356 s. ipotizza che, partendo da questo spunto indiretto, Silio abbia elaborato la scena successiva con l'apparizione onirica di Minerva a Diomede.

40. Serv. ad Aen. 2, 166 transeunti per Calabriam.

41. Contra, ad Aen. 3, 550, leggiamo che fu direttamente Enea a riceverlo a Diomede vel ab Ulixe; anche Festo 422 L., a proposito dell'usanza di sacrificare velato capite, riporta un aneddoto relativo al mancato incontro tra Enea, impegnato nel rito, e Ulisse.

42. La colpa commessa è il ratto del Palladio, rievocato ai vv. precedenti (36-50) in una sezione largamente indebitata con il secondo libro dell'Eneide (Ripoll 2001, 358 ss.). 
43. Cfr. anche 55 Laomedonteae... Minervae; 78 Troianam... Minervam. Sulla relativa ambiguità e le possibili implicazioni dell'epiteto Phrygius, cfr. più oltre.

44. Aen. 11, 271 nunc etiam horribili visu portenta secuntur. L'espressione sembrerebbe alludere proletticamente alle prodigiose metamorfosi dei compagni in uccelli, descritte di seguito, e comunque riferirsi a manifestazioni dell'ira di Venere, la divinità irata contro il Diomede dell' Eneide (Horsfall 2003, 187 ad Aen. 11, 271). Tuttavia l'idea di "apparizioni terribili e persecutrici" che ancora assillano Diomede (vittima del passato 'che ritorna') potrebbe aver suggerito a Silio ('collaborando' con la notizia varroniana testimoniata da Servio ad Aen. 2, 166, cfr. sopra, n. 39) l'idea della successiva scena onirica con protagonista Minerva.

45. Comunque efficace, a questo proposito, l'analisi di Ripoll 2001, 365.

46. A differenza di Minerva, così definita in modo abbastanza singolare in Sil. 13, 52 s. (cfr. sopra, nel testo e alla n. 43).

47. La scena in questione trova un pendant verso la fine delle Metamorfosi (15, 653 ss.), quando Esculapio appare in sogno a un cittadino romano (probabilmente Ogulnio), annunciandogli questa volta il suo arrivo nella nuova sede.

48. Cfr. in part. von Albrecht 1968. Sulla presenza dei Fasti nei Punica cfr. Santini 1983.

49. È l'esortazione di Apollo (ancora lui) ai discendenti dei Troiani. Poco prima erano risuonate le parole di un altro oracolo (quello trascritto dai libri sibillini, consultati per l'occasione): fast. 4, 259 "Mater abest: Matrem iubeo, Romane, requiras" (Fantham 1998, 146 s. ad l.).

50. Phryx è "more than a gloss for pius Aeneas" (Fantham 1998, $150 \mathrm{ad}$ l.).

51. Per la presenza del modello di Aen. 6, 489 ss. (negli inferi le ombre dei Greci tremano alla vista di Enea in armi), cfr. Ripoll 2001, 364 s. Naturalmente il 'ritorno del passato' è un fenomeno che possiede implicazioni precise anche per un testo come i Punica, che vede riemergere con forza (dal proprio passato storico-letterario) la sagoma potente e autorevole dell'Eneide.

52. A differenza del suo alter ego virgiliano, il Diomede di Silio non è pentito di aver ferito Venere in battaglia (Aen. 11, 275 ss.), quanto piuttosto esprime il rammarico di esser stato indotto dal destino e dal volere degli dèi a partecipare alla guerra di Troia.

53. Su una nave arrivano a Roma anche Cibele (in Ovidio e poi in Silio) ed Esculapio (in Ovidio).

54. Anche Ripoll $(2001,361)$ è costretto a ipotizzare un suggerimento 'trasversale': la punizione dei Galli sarebbe una riattualizzazione di quella dei Greci reduci da Troia. Da notare, en passant, che la notizia fornita da Dasio costituisce l'ultimo frammento della rievocazione siliana dell'assalto portato dai Galli a Roma (cfr. sopra).

55. Cic. Scaur. 48; Liv. 5, 52, 7 e 26, 27, 14; Dion.Hal. Ant.Rom. 2, 66, 4; Ov. fast. 1, 527 s. e 6, 427 ss.; Serv.Dan. ad Aen. 2, 188. Per il trasferimento a Caere dei sacra custoditi nel tempio di Vesta, in occasione dell'arrivo dei Galli alle porte di Roma, cfr. Liv. 5, 40, 7-10 e Ogilvie 1965, 723 ss. ad l.

56. Se le parole di Sinone $(2,169-175)$ possono comunque suscitare nel lettore qualche dubbio sulla loro affidabilità (ma cfr. sopra, nel testo), è necessario ricorrere al racconto di Diomede (11, 255 ss.), che peraltro è assai meno esplicito nel collegare il ratto del Palladio all'ira della dea contro i Greci.

57. Malgrado la rassegnata decisione di ritirare le truppe dall'assedio di Roma, Annibale non rinuncia a ribellarsi al destino neppure più tardi, quando è già imbarcato sulla nave che lo riporta in Africa (a precludergli ogni possibilità di riapprodare in Italia è una tempesta scatenata da Nettuno: 17, 236 ss.). L'amaro presentimento dello scacco finale, che pure inizia a farsi strada nell'animo del personaggio, non trova in Silio un'espressione diretta paragonabile alle parole che gli erano state attribuite da Orazio dopo la sconfitta di Asdrubale al Metauro (carm. 4, 4, 53 ss.). Per il suo forte valore paradigmatico quest'ultimo confronto, segnalatomi dall'anonimo referee della rivista, merita comunque notevole attenzione (un caso interessante di 'impronta oraziana' è, per es., anche la descrizione di Regolo prigioniero in Sil. 2, 340 ss., da confrontare con Hor. carm. 3, 5, 18 ss.; Fucecchi 2003, 278). 


\section{RIASSUNTI}

L'ampia narrazione della campagna militare di Annibale in Italia offre a Silio Italico l'opportunità di aprire alcune finestre sulla precedente storia di Roma, in forma di ekphraseis e racconti inseriti. Alcuni momenti del passato più o meno recente (la prima guerra punica e l'invasione della città da parte dei Galli, all'inizio del IV sec. a. C.) vengono illustrati mediante una dialettica di interpretazioni contrapposte, che conferisce alla guerra in atto i connotati aggiuntivi di una lotta per il controllo del passato stesso. La rievocazione di episodi ancora più antichi e ammantati di leggenda (il sacrificio dei Fabii, l'impresa di Clelia, fino alla restituzione del Palladio a Enea da parte di Diomede) sembra, viceversa, assumere un ruolo specifico nella dinamica del conflitto: quello di contrastare l'avanzata apparentemente inarrestabile del condottiero cartaginese e di vanificarne, infine, il progetto di conquistare il Campidoglio. Da un altro punto di vista, l'attualizzazione 'esemplare' della storia di Roma impone un confronto critico con la tradizione e riflette il modo in cui i Punica ricostruiscono la propria genealogia letteraria.

\section{INDICE}

Mots-clés : Epica flavia, Storia romana, tecnica narrativa, ekphrasis, metadiegesi, intertestualità, poesia augustea

\section{AUTORE \\ MARCO FUCECCHI}

Università di Udine 